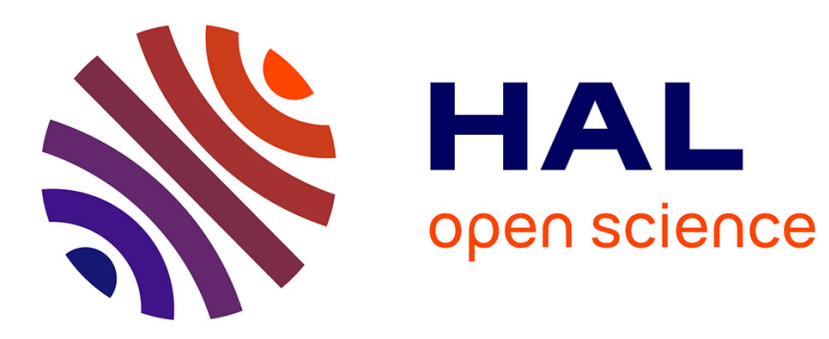

\title{
Vers un modèle hydrologique simplifié pour les études géomécaniques spatialisées
}

Gilles Grandjean, Vincent Mardhel, Audrey Hohmann, Rosalie Vandromme, Olivier Sedan

\section{- To cite this version:}

Gilles Grandjean, Vincent Mardhel, Audrey Hohmann, Rosalie Vandromme, Olivier Sedan. Vers un modèle hydrologique simplifié pour les études géomécaniques spatialisées. La Houille Blanche - Revue internationale de l'eau, 2010, 3, pp.53-57. 10.1051/lhb/2010034 . hal-00540182

\section{HAL Id: hal-00540182 https: / hal-brgm.archives-ouvertes.fr/hal-00540182}

Submitted on 26 Nov 2010

HAL is a multi-disciplinary open access archive for the deposit and dissemination of scientific research documents, whether they are published or not. The documents may come from teaching and research institutions in France or abroad, or from public or private research centers.
L'archive ouverte pluridisciplinaire HAL, est destinée au dépôt et à la diffusion de documents scientifiques de niveau recherche, publiés ou non, émanant des établissements d'enseignement et de recherche français ou étrangers, des laboratoires publics ou privés. 


\title{
Vers un modèle hydrologique simplifié pour les études géomécaniques spatialisées.
}

\author{
G.Grandjean ${ }^{1}$, V.Mardhel, A.Hohmann, R.Vandromme \\ BRGM, Orléans, France
}

\begin{abstract}
Estimating soils geomechanical behavior interest numerous applications dealing with natural hazards, landscape management or terrestrial vehicles mobility. These applications need most of the time high time reactivity in producing decision-making maps, a strong input data availability and a spatial resolution in the order of decametric scale. This work presents an simplified hydrologic model allowing estimation of soil moisture from a generic dataset. This model is based on qualitative indexes related to runoff and infiltration processes that are convolved with climatic data extracted from a global database. The link between the resulting moisture map and the soil geomechanical behavior can be established from field observations or laboratory measurements. This model is applied to two different cases history and is discussed in term of positive results and limitations.
\end{abstract}

Résumé - L'estimation du comportement géomécanique des sols ou du proche sous-sol rentre dans bon nombre d'applications dédiées aux risques naturels, à l'aménagement du territoire ou à la mobilité des véhicules terrestres en tout-terrain. Ces applications nécessitent souvent une forte réactivité dans la production des diagnostics, l'utilisation de données facilement accessibles et une résolution de l'ordre du décamètre. Ce travail présente un modèle hydrologique simplifié permettant d'estimer rapidement un indice d'humidité des sols à partir d'un jeu de données générique. Le modèle est basé sur le calcul d'indices qualitatifs - rendant compte respectivement des effets de ruissellement et d'infiltration - qui sont ensuite pondérés par des données climatologiques issus de bases de données globales. Le lien vers le comportement géomécanique des sols est réalisé grâce à des abaques d'évolution de la portance des sols en fonction du taux d'humidité ou grâce à des observations in situ. Les deux applications présentées montrent l'efficacité, mais aussi les limites, du modèle développé pour la production de carte de mobilité de véhicules ou de cartes de susceptibilité aux mouvements de terrain.

\section{Introduction}

La teneur en eau est un facteur déterminant quand il s'agit d'estimer le comportement des sols d'un point de vue géomécanique. Que se soit pour estimer l'ampleur des mouvements relatifs aux argiles gonflantes, la stabilité des versants altérés ou la portance des surfaces de roulements, la relation entre les diverses propriétés du sol et la saturation en eau est clairement admise, souvent assez bien décrite physiquement, et parfois mesurée localement lors d'expérimentations in situ. En revanche, peu de modèles offrent la possibilité de spatialiser ces connaissances afin de pouvoir appréhender le phénomène aux échelles régionales. Pourtant, les problématiques d'aménagement du territoire et d'estimation des aléas relatifs à l'état de la proche surface passent par des études phénoménologiques à ces échelles.

\footnotetext{
${ }^{1}$ g.grandjean@brgm.fr
} 
En outre, une résolution spatiale fine est souvent requise pour que le diagnostic soit opportun. Par exemple, il peut s'agir de localiser les points kilométriques d'une route qui seront affectés par des éboulements, les bâtiments qui seront effectivement atteints par un affaissement ou le meilleur itinéraire pour un véhicule devant naviguer en tout-terrain sans risquer de s'enliser.

Le travail présenté a pour objectif d'estimer un indice d'humidité qui reflètera qualitativement la teneur en eau du sol sur des emprises de l'ordre de plusieurs dizaines de kilomètres à des résolutions décamétriques. Le principe repose sur l'assimilation de plusieurs types de données : W la nappe d'eau issue de statistiques climatologiques globales, TWIC un indice de ruissellement prenant en compte la morphologie du terrain depuis les échines vers les rivières, IDPR un indice d'infiltration indiquant les endroits où l'eau est préférentiellement drainée du sol vers le sous-sol ; la contribution entre ruissellement et infiltration étant donné par le type de sol défini selon la norme USCS. L'algorithme développé consiste à faire un bilan qualitatif entre l'eau qui est amené par la pluie corrigée des effets d'évapotranspiration, l'eau qui ruisselle vers les points bas et l'eau qui s'infiltre vers le sous-sol.

Ce modèle est utilisé dans le cadre de deux applications : l'une vise à estimer la mobilité des véhicules sur des sols meubles en fonction du taux d'humidité estimé à une saison donnée (Projet d'Etude Amont ECORS, Grandjean et al., 2008), l'autre a pour objectif de délimiter les zones les plus propice aux éboulements le long d'une route de Guadeloupe afin d'implanter un système de surveillance et de mettre en place à terme une méthode de prévision des évènements de grande ampleur (Mompelat, 1994).

\section{Indice d'Humidité Qualitatif}

De nombreux travaux présentent des solutions pour estimer l'humidité des le proche sous-sol. Ces études utilisent des techniques d'assimilation de données pluies-débit (Weisse et al., 2003), des données satellitaires (Wagner et al., 1999, Glenn and Carr, 2004, Baghdadi, 2007) ou des modèles de prédiction hydrologiques (Ducrocq et al., 2004). La plupart de ces méthodes, complexes à mettre en œuvre, donnent une indication quantitative de l'humidité car elles font appel à des modèles physiques calés sur les bilans météo-hydrologiques ou des données de calibration au sol.

Dans le cadre d'une approche simplifiée du problème, où seulement la gestion des variations relatives de l'humidité des sols est nécessaire, nous proposons d'utiliser un indice basé uniquement sur la réponse du sol à un évènement pluvieux. Cet indice tire parti d'un certain nombre de travaux qui ont étudié ce genre de réponse et sa représentation spatiale :

- Les écoulements de surface: modélisé par un indice d'humidité topographique (Topographic Wetness Index, TWI) selon Beven and Kirby (1979) ;

- Les phénomènes d’infiltration: modélisé par le calcul de l'IDPR (Indice de persistance et de Développement des Réseaux ; Mardhel et al., 2004) ;

Dans notre démarche, le bilan hydrologique estime le volume d'eau qui tend à s'infiltrer localement dans le sol à partir du réservoir pluviométrique - corrigé de l'évapo-transpiration, EVT. Ce réservoir doit être diminué de l'eau qui s’écoule en aval par ruissellement, augmenté de l'eau qui arrive de l'amont par ruissellement, et diminué de l'eau qui s'infiltre dans les couche basses du sous-sol. La méthode permettant de combiner ces phénomènes, réside alors 
simplement dans une simple pondération entre les indices morphologiques TWI, IDPR et donnée d'entrée climatologique, comme l’indique la Figure 1.

L'avantage de cette méthode provient du fait qu'elle utilise des variables spatialisées à l'échelle du MNT, considéré à $30 \mathrm{~m}$ dans nos travaux. La climatologie est issue de la base globale GLDAS qui renseigne sur l'état hydrique (corrigé de l'EVT) de la couche superficielle du sous-sol. Le calcul de l'IDPR nécessite d'intégrer le réseau hydrologique pérenne qui est ensuite comparé au réseau de ruissellement donné par le MNT. L'état de saturation face à l'humidité du sol calculé à l'échelle locale est ensuite estimé par un index de saturation (Soil Water Holding Capacity, SWHC; http://gcmd.nasa.gov/records/ GCMD_GNV0025.html) dépendant de la nature du sol selon la classification internationales USCS des sols.

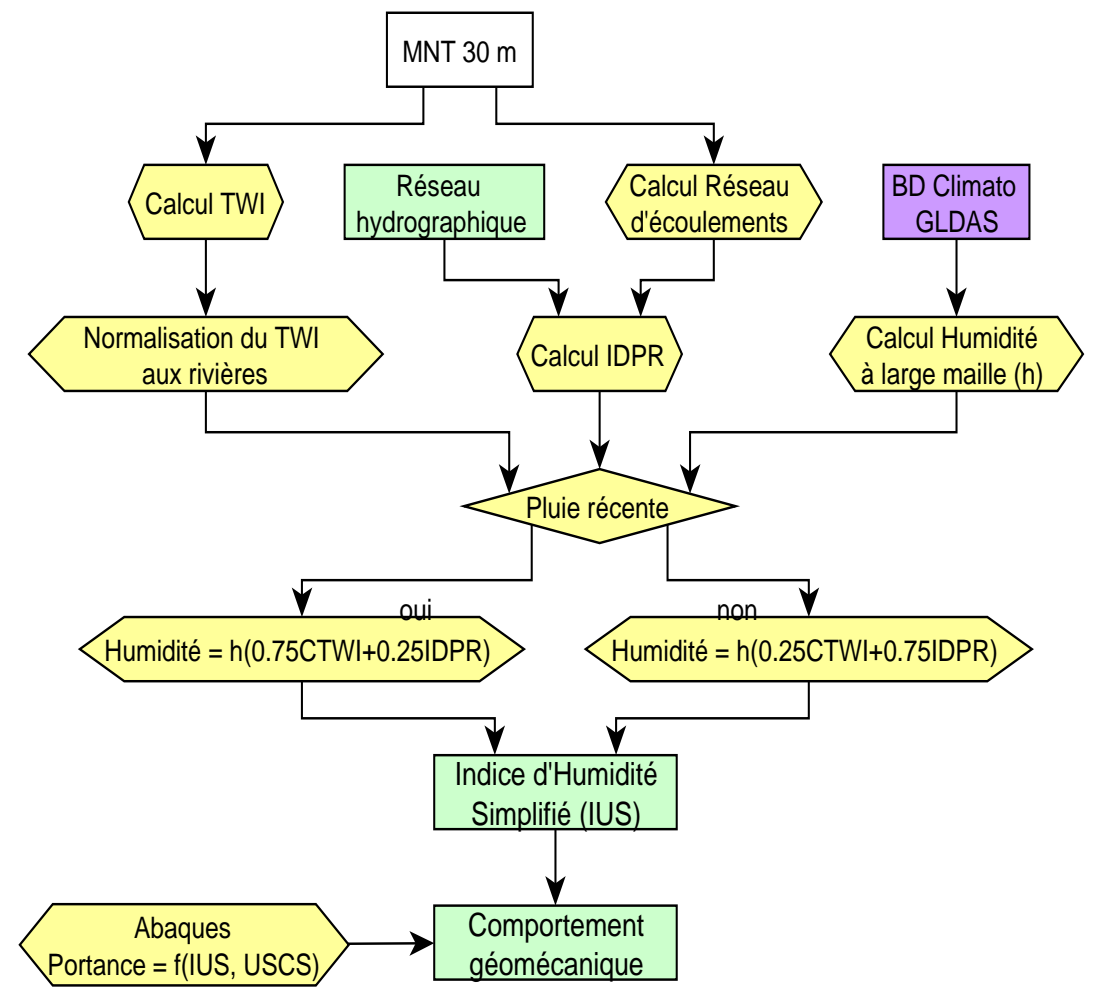

Figure 1 : flux de traitement de l'indice simplifié d'humidité.

\section{Cas d'étude 1 : aide à la décision pour la mobilité (PEA ECORS)}

Cette étude décrit le système d’aide à la décision ECORS dédié à la caractérisation des routes et des sols pour la mobilité et la projection des forces en contexte opérationnel. Ce projet, initié par le DGA (Délégation Générale à l'Armement), a été confié à un groupement d'industriels, de sociétés d'ingénierie et de laboratoires de recherche pour sa réalisation technique. L'objectif d'une telle étude est tout d'abord d'analyser et d'évaluer les technologies disponibles - actuellement et à moyens termes - pour la caractérisation des routes, des pistes et des sols pour la mobilité des véhicules, que ce soit en territoire français ou en théâtres d'opérations extérieurs. Les principales sources autorisées par les exigences du projet comprennent toutes les données disponibles de façon générique et globale permettant d'apporter de l'information sur le théâtre d’opération à distance de sécurité. Les données identifiées sont les images de télédétection, les bases de données globales telles que Topobase-Géobase, Corrine Landcover, GLDAS et toute donnée open-source pertinente. Une 
fois acheminée dans le système, ces données sont gérées par un outil de calcul basé sur un Système d’Information Géographique (SIG). Ce dernier calcule alors les capacités d'un véhicule de se mouvoir sur un terrain donné identifié sur le théâtre. Cette opération de calcul est réalisée en comparant les caractéristiques du terrain (pente, occupation du sol, humidité, rugosité, nature du sol) à des cas réels ou simulés et stockés dans une base de données. L'estimation des vitesses peut donc être réalisée suivant plusieurs scénarii climatiques, comme le montre la Figure 2.

On notera que la vitesse est fortement corrélée à l'humidité, même si ce paramètre n'est qu'une composante de la mobilité avec l'occupation du sol, la pente la rugosité du terrain et la nature du sol.
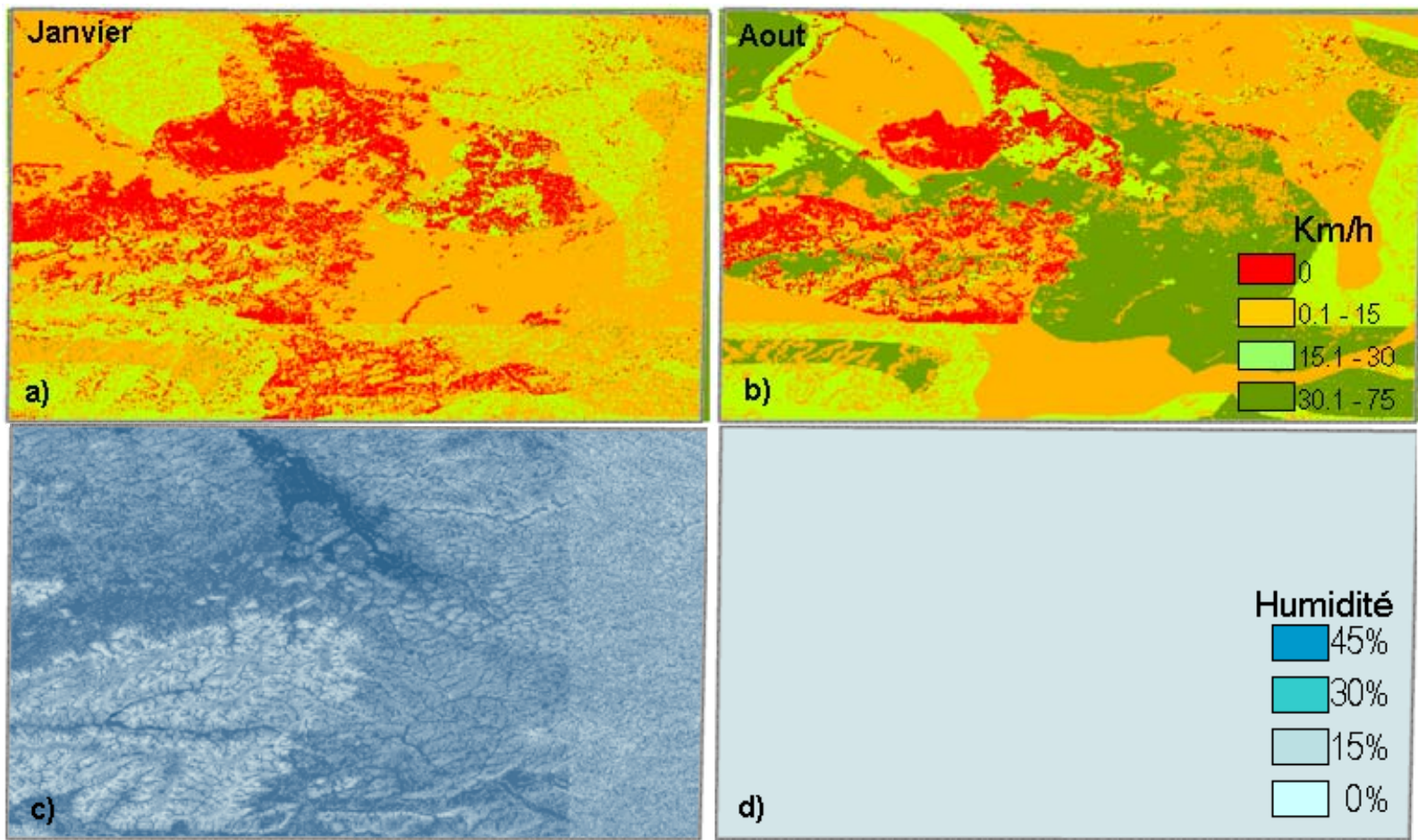

Figure 2 : carte d'humidité (e, f) et estimation des vitesses de progression associées (a, b) pour un véhicule de type $4 \times 4$ à deux saisons différentes.

\section{Cas d'étude 2 : carte de susceptibilité aux mouvements de terrain en Guadeloupe}

Pour ce cas d'étude, il s'agit d'appréhender le comportement des versants qui longent la route de Mamelles afin d'identifier à priori les zones susceptibles aux mouvements de terrain. Dans cette région, fortement soumise aux évènements cycloniques, l'apport en eau dans les couches altérées de la proche surface est l'un des critères majeurs, avec la pente, qui peuvent déclencher des glissements de terrain. Suite à une reconnaissance sur site fin 2008, une soixantaine d'évènements ont été recensés.

Le modèle d'indice d'humidité simplifié a été utilisé pour estimer une saturation en eau, la pente locale a été extraite des données du MNT IGN à $50 \mathrm{~m}$, la nature du sol reconnue à partir des relevés géologiques. La Figure 3 montre, le long de la route, l'état du MNT, de l'IHS, ainsi que la carte de susceptibilité résultante. Le modèle utilisé pour quantifier la susceptibilité a consisté à analyser statistiquement ces paramètres afin d'identifier les situations les plus probables conduisant à un glissement. La population d'’instabilités indiquée sur le graphe de la Figure 3 montre une très nette tendance qui a été modélisée par une droite. Sont considérés 
comme instables les emplacements dont les propriétés en termes de pente et d'humidité se situent au-delà de la droite.
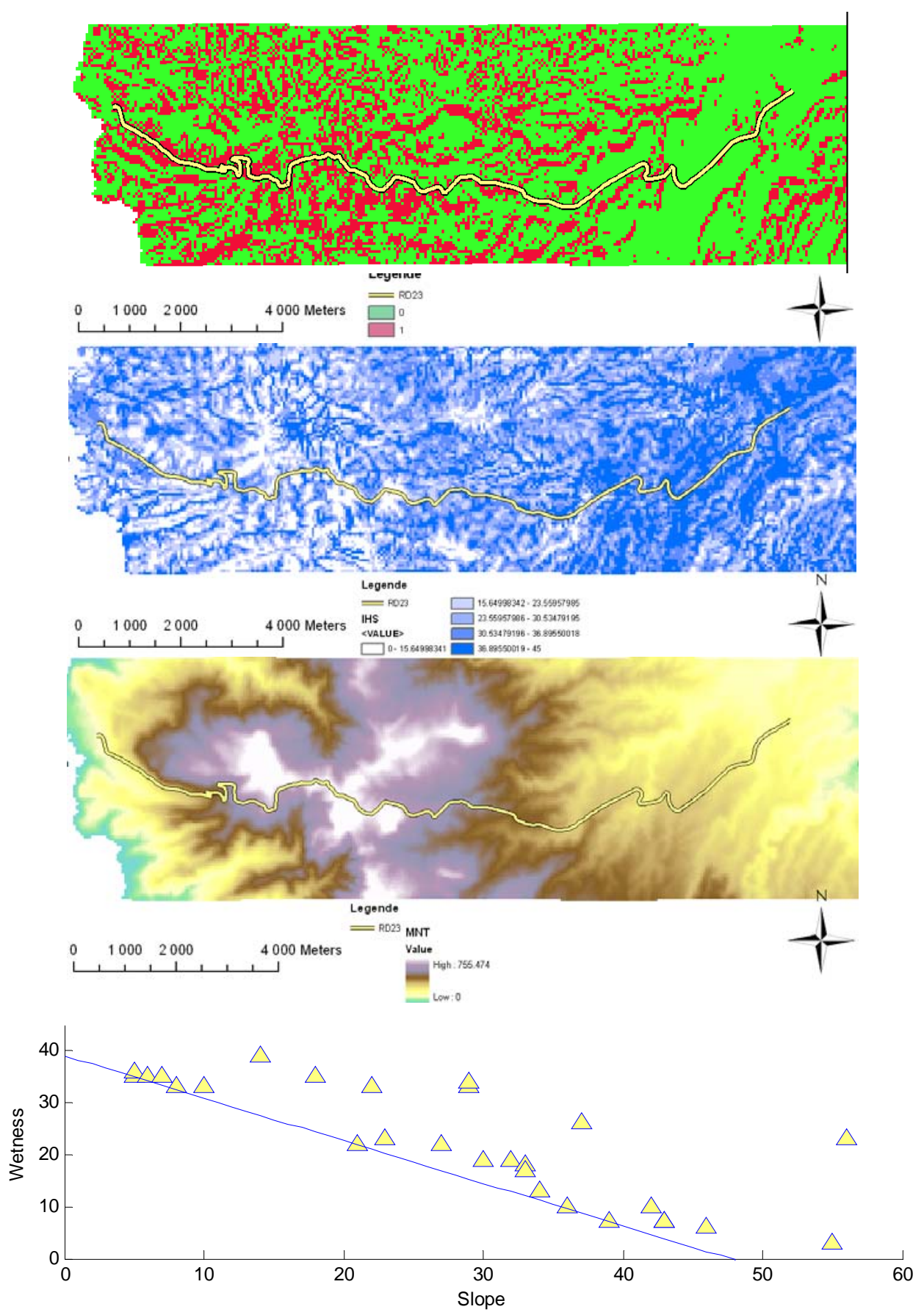

Figure 3 : carte d'humidité, des pentes et de la nature du sol le long de la route des Mamelles (Guadeloupe)

\section{Conclusions}

Ce travail a présenté un modèle hydrologique simplifié permettant d'estimer rapidement un indice d'humidité des sols à partir d'un jeu de données générique. Le modèle est basé sur le calcul d'indices qualitatifs mais spatialisés - rendant compte respectivement des effets de ruissellement et d'infiltration - qui sont ensuite pondérés par des données climatologiques issus de bases de données globales. Le lien vers le comportement géomécanique des sols est 
réalisé grâce à des abaques d'évolution de la portance des sols en fonction du taux d'humidité, ou grâce à des relevés in situ.

Deux applications montrent l'utilité d'intégrer un tel indice dans les estimations de mobilité de véhicules terrestres ou d'estimation de cartes de susceptibilité aux mouvements de terrain. Même si l'indice simplifié d'humidité ne reste qu'une estimation qualitative de la teneur en eau dans le sol, il offre des avantages non négligeables : généricité des données sources qui peuvent être approvisionnées rapidement et de façon globale, rapidité des calculs, bonne résolution liée à celle du MNT utilisé. Les perspectives de ce travail vont consister à identifier un moyen de calibrer la carte de l'indice d'humidité produite à partir de données météo observées.

\section{Remerciements}

Ces travaux ont été financés par le BRGM et la DGA au travers du PEA-ECORS. Les auteurs remercient P.Mompelat, O.Sedan pour leur contribution à la connaissance de la Route de Mamelles.

\section{Bibliographie}

Baghdadi, N., Aubert, M., Cerdan, O., Franchistéguy, L., Viel, C., Eric, M., Zribi, M., Desprats, J.F., 2007. Operational Mapping of Soil Moisture Using Synthetic Aperture Radar Data: Application to the Touch Basin (France). Sensors 2007, 7, 10, 2458-2483.

Beven, K. J. and Kirkby, M. J.: A physically based, variable contributing area model of basin hydrology, Hydrol. Sci. Bull., 24, 43-69, 1979.

Ducrocq, V., F. Bouttier, S. Malardel, T. Montmerle et Y. Seity, 2005 : Le projet AROME, Crues Méditerranéennes : Les réponses scientifiques et techniques de l'Etat. La Houille Blanche, 2, 39-44.

Glenn, N.F. and Carr, J.R., 2004. Establishing a relationship between soil moisture and RADARSAT1 SAR data obtained over the Great Basin, Nevada, U.S.A., Can. J. Remote Sensing, Vol. 30, No. 2, pp. 176-181.

Grandjean, G. Grima, M. and ECORS Team, 2008. The ECORS system: a mobility decision-making tool. 16th Int. Conf. Int. Soc. Terrain-Vehicle Systems - Turin November.

Mardhel V., Frantar P., Uhan J., Mio A., 2004. Index of development and persistence of the river networks as a component of regional groundwater vulnerability assessment in Slovenia. .Int. Conf. groundwater vulnerability assessment and mapping. Ustron, Poland, 15-18 June 2004., Poland.

Mompelat, P., 1994. Unités cartographiques et évaluation de l'aléa mouvements de terrain en Guadeloupe (Antilles françaises). Thèse Univ. Paris, France, 337p.

Wagner, W., G. Lemoine, H. Rott, 1999. A method for estimating soil moisture from ERS scatterometer and soil data, Remote Sensing of Environment, 70, 191-207

Weisse, A., Oudin, L., Loumagne, C., 2003. Assimilation de données d'humidité des sols pour la prévision de crues : Comparaison d'un modèle pluie-débit conceptuel et d'un modèle intégrant une interface sol-végétation-atmosphère. Revue des sciences de l'eau, 16, 2, 173-197. 